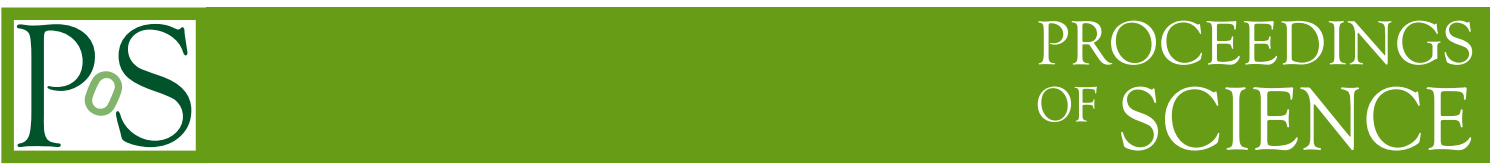

\title{
The Study of PWNe with a photoelectric polarimeter
}

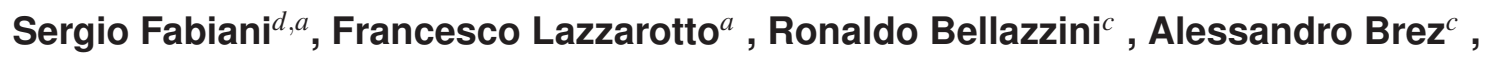 Enrico Costa $^{a}$, Sergio Di Cosimo ${ }^{a}$, Fabio Muleri $^{a, b}$, Alda Rubini $^{a}$, Paolo Soffitta ${ }^{a}$, Gloria Spandre ${ }^{c}$.}

${ }^{a}$ Istituto di Astrofisica Spaziale e Fisica Cosmica

Via del Fosso del Cavaliere 100, I-00133 Roma, Italy

${ }^{b}$ Università di Roma Tor Vergata, Dipartimento di Fisica

Via della Ricerca Scientifica 1, 00133 Roma, Italy

${ }^{c}$ Istituto Nazionale di Fisica Nucleare

Largo B. Pontecorvo 3, I-56127 Pisa, Italy

${ }^{d}$ Università di Roma La Sapienza, Dipartimento di Fsica

Piazzale Aldo Moro 5, 00185 Roma, Italy

E-mail: sergio.fabiani@iasf-roma.inaf.it

The measurement of the polarization of Pulsar Wind Nebulae is a powerful tool to investigate the flow structure of the wind produced by the central pulsar and the magnetic field in the nebula. Unfortunately, polarimeters in the X-ray range where these sources are easily observed are not available yet. However, the recent development of instruments based on photoelectric absorption is opening the possibility to observe a large number of PWNe with sufficient sensitivity to measure a degree of polarization at the level of $\%$ and resolve the closer sources. Here we present the first simulations of the angular resolution of the Gas Pixel Detector, one of the most advanced project in the field of photoelectric polarimeters. We assume that it will be placed in orbit in the context of a pathfinder mission, like POLARIX, or in the focal plane of a large mission like the International X-ray Observatory (IXO).

Polarimetry days in Rome: Crab status, theory and prospects October 16-17 2008

Rome, Italy 


\section{Introduction}

It is expected that the Pulsar Wind Nebulae emit strongly polarized radiation because of particles accelerated by the central pulsar produce synchrotron emission in the magnetic field of the nebula. The prototype of these sources, the Crab Nebula, emits X-ray emission which is about $20 \%$ polarized at a few $\mathrm{keV}$, as measured by the polarimeter on-board OSO-8 (Weisskopf 1978). Recent MHD relativistic simulations highlight that space-resolved measurements of the direction and of the degree of polarization allow to study the velocity of the wind and the magnetic field in the nebula. This will probe the formation of the jet and the presence of non-toroidal component of the magnetic field in the nebula (Bucciantini 2005), (Del Zanna 2006) and (Nakamura 2007). Thanks to recent observations we know that Crab and other PWNe have a similar structure in X-ray band. They are characterized by thin structures such as arcs, rings, the central knot and possibly the presence of one or two jets apparently coming from the inner region close to the pulsar (Weisskopf 2000). Another remarkable feature in X ray band is the presence of an emitting equatorial torus, associated to a high magnetized region. Equatorial wind flows from the central pulsar and the formation of the termination shock explain the formation of these structures (Del Zanna 2006). The on axis component of the field is linked to the emission of jets, whereas the equatorial magnetization is responsible for the brightness of the torus. The magnetic field of pulsar wind is toroidal far beyond the light cylinder because of the rotation, however disordered components are expected to be. This components could be an indicator for transfer energy from toroidal magnetic field to plasmas (Nakamura 2007).

Polarization measurement are crucial to predict polarization maps that could be tested by future observations. For example Figure 1 reports a simulation for the optical polarization degree (on left) and the surface of brightness (on right) with overlapped ticks whose direction is that of the polarization and whose size is proportional to the fraction of the polarization. This figure shows a
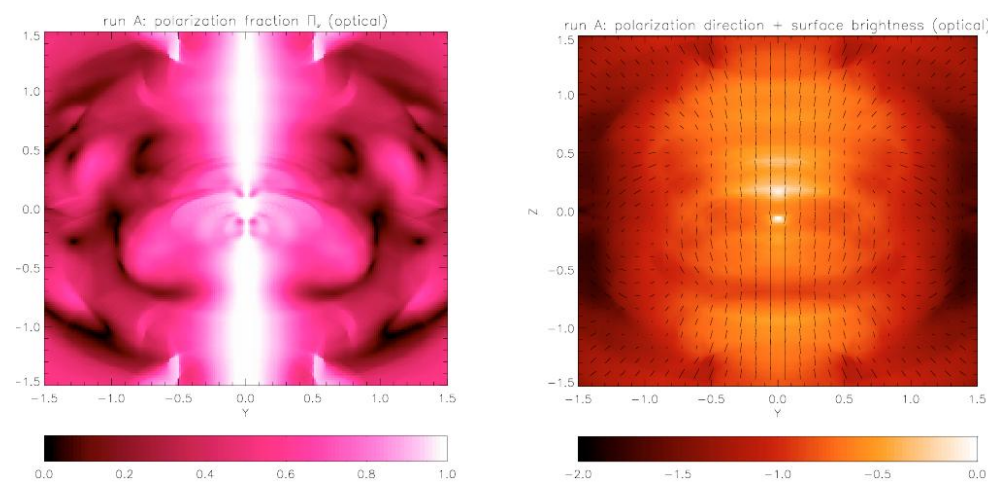

Figure 1: Maps for simulations of optical polarization normalized at the degree of about 70\%. On left: Map of polarization degree. White regions are characterized by a high degree of polarization (up to 70\%). On right: surface of brightness with overlapped ticks whose direction is parallel to the direction of polarization and whose size is proportional to the polarization degree. (Del Zanna 2006)

complex structure of the polarization arising from the the complexity of the field and the overlapping of opposite regions projected in front of the observer. High degree of polarization is expected 
(up to $70 \%$ ). It is clear that the ability to resolve the structure of polarization is crucial to derive experimental maps to compare with theoretical expectations.

\section{Gas Pixel Detector}

The Gas Pixel Detector is essentially a gas detector which is able to image the photoelectron tracks. It exploit the dependence of photoelectric cross section to photon polarization direction to the aim of measuring polarization (Bellazzini 2007). In the GPD photons are absorbed by photoelectric effect in a gas cell filled with a gas mixture. The photoelectron is emitted with more probability in the direction of the electromagnetic field of the photon. As the photoelectron propagates, it loses its energy by ionization and it is scattered by charges in the nuclei. The generated electron-ion pairs, which trace the photoelectron path, are drifted and amplified by the Gas Electron Multiplier (GEM) and eventually collected on a fine sub-divided pixel detector. The projection of the track on the plane of the detector is analyzed and the direction of ejection of the photoelectron is reconstructed together with the absorption point. Changing the components of the gas mixture

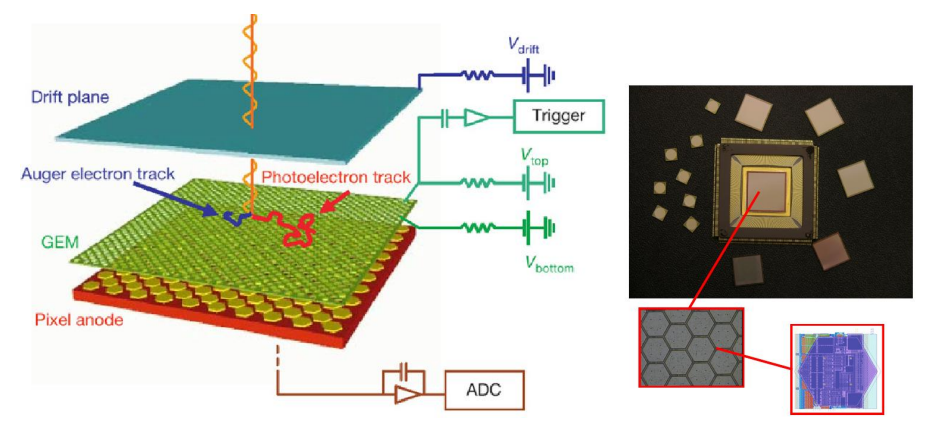

Figure 2: On left: The scheme of GPD. It is illustrated the absorption of a photon and the production of the photoelectron together with an Auger electron. On right: Some image of the anode plane and of its hexagonal pixels.

it is possible to select properly the energy band of employment of the instrument in the range of about $1 \div 30 \mathrm{keV}$. This is the wide band in which the photoelectric absorption is effective in gas. However currently the instrument is optimized in the $2 \div 10 \mathrm{keV}$ energy band.

The further aspect of this detector is the capability to preserve the imaging while reaching a good sensitivity in polarization as well as in spectroscopic and timing measurements.

The current detector is $15 \times 15 \mathrm{~mm}^{2}$ and weights only $50 \mathrm{~g}$. It is the result of three subsequent generations. At present the chip integrates more than 16.5 million transistors. It has an active area of $105^{\prime} 600$ pixels organized in a honeycomb matrix 300x352. It is a self triggered system able to select itself the pixels region interested by a track.

\section{Effect of the optics}

The employment of a X-ray optics is mandatory to collect the large number of photons needed to perform a polarimetric study at the \% level. The photons are not absorbed in an infinitely thin focal plane but in a gas cell $1 \mathrm{~cm}$ thick (see fig.3), than the focusing of the radiation causes an 
additional blurring which sums with the PSF of the optics. Usually X-ray telescope optics are composed by shells. Every one of whitch is formed by a paraboloid and a hyperboloid surfaces. Reflection of X-rays is due to grazing incidence on shells surface and the collecting area of every shell depends on photons energy.

We have developed a simple software that allows to simulate the absorption in gas of the photons reflected by the optics. Photons experience two reflections on the two shells surfaces, the first of which with a grazing angle $\alpha$. Eventually they arrive in the gas cell with an inclination angle of $4 \alpha$ with respect to the focal axis, that in this case is coincident with the normal to the window surface (see Fig. 3 left panel).
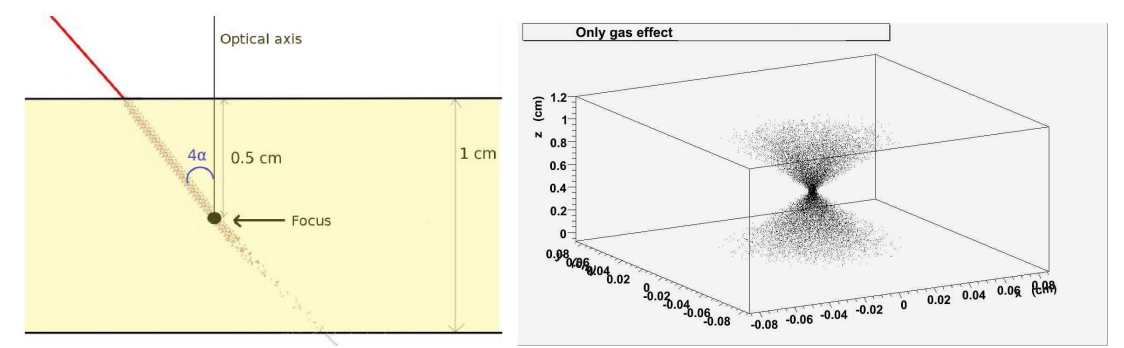

Figure 3: On left: The scheme of the absorption of an inclined ray. On right: Effect of photon inclined penetration and absorption in gas with an ideal perfect optics system for the POLARIX case. Photons at the energy of $3 \mathrm{keV}$ are absorbed in a gas mixture composed by 70\% of DME and 30\% of He at the pressure of 1 atm and $1 \mathrm{~cm}$ thick.

Firstly we calculate the lenght of the effective photon path in gas. We derive the absorption point from the angle $4 \alpha$ between the focal axis and the direction of incoming photon. The values of $\alpha$ are tabulated in a file. We generate the absorption coordinates $\left(x_{\text {gas }}, y_{\text {gas }}\right)$ associating to the separation radius of the absorption point from the focal axis an angle $\phi$ uniformly distributed in the interval $[0,2 \pi]$. The software allows to perform a resolution analysis computing the $50 \%$ (Half Power Width (HPW) ) and 90\% containment diameters of absorption counts around the focal axis. In this way we can obtain the effect of absorption in gas on inclined photon penetration as coming from an ideal perfect optics system.

It could be possible to add in input to this tool a ray-tracing software that fully takes into account the physics of polarized X-ray reflection on shells. For the moment we simply try to derive the magnitude of the effect of the absorption in gas and of the PSF of the optics. We consider an usual parametrization of X-ray PSF on the focal plane. Its section is described with the equation (3.1) (King profile):

$$
K P(\theta)=\left(1+\left(\frac{\theta}{c_{2}}\right)^{2}\right)^{-c_{3}}
$$

where $\theta$ is the angular separation from the peak profile and the parameter $c_{2}$ is to be expressed in arcsec unit, whereas $c_{3}$ is a dimensionless number. The integral, on the focal plane, of the profile is given by the equation (3.2):

$$
I K(\theta)=\pi \frac{c_{2}^{2}}{1-c_{3}}\left[\left(1+\left(\frac{\theta}{c_{2}}\right)^{2}\right)^{1-c_{3}}-1\right]
$$


whose normalization constant is:

$$
I K_{\theta \rightarrow \infty}^{1-c_{3}<0}=\pi \frac{c_{2}^{2}}{c_{3}-1}
$$

The ratio between the Eq. 3.2 and the Eq. 3.3 is a random number $R$ in the interval $[0,1]$. Than we can obtain the angular separation $\theta$ simply by inverting the relation given by this ratio. The $\theta$ distribution is than:

$$
\theta=c_{2} \sqrt{(1-R)^{1 /\left(1-c_{3}\right)}-1}
$$

We can translate this angular separation in space separation on the focal plane by multiplying $\theta$ for the focal length of the optics. In this way for every absorption event we can generate as before $\left(x_{\text {opt }}, y_{\text {opt }}\right)$ coordinates to sum to the $\left(x_{\text {gas }}, y_{\text {gas }}\right)$ already computed. So we are able to consider one by one the two effects on resolution: the effect of inclined penetration in the gas cell and the effect of PSF. Moreover we are able to make the sum of this two effects simply by summing linearly the coordinates.

We perform this simulation in two possible scenarios. One for an optics with a focal length of 3.5 meter. This could be the case of the low-cost pathfinder Italian mission POLARIX (currently in phase A study by the Italian Space Agency). The second scenario is that of a large mission like the International X-ray Observatory (IXO). It currently includes a GPD in its focal plane. We consider one of the possible options with the focal length of 20 meter. Our results are shown in Tab. 1. They indicate that the HPW given by the gas blurring is about the $40 \%$ to the total angular resolution.

\begin{tabular}{ccccc}
\hline POLARIX & Energy (keV) & HPW gas+optics (") & HPW optics (") & HPW gas (") \\
\hline & 2 & 24 & 18 & 12 \\
\hline & 3 & 24 & 18 & 12 \\
\hline \hline IXO & 6 & 22 & 18 & 10 \\
\hline & 2 & & & 3 \\
\hline & 3 & 6 & 5 & 2 \\
\hline & 6 & 6 & 5 & 1 \\
\hline
\end{tabular}

Table 1: Simulations of the HPW for POLARIX and IXO scenarios performed at different energies taking into account the gas effect, the optics effect and their combination.

\section{Conclusions}

The sensitivity of the GPD is sufficient to detect X-ray polarization from many PWNe (see Table 2). If we take into account the Minimum Detectable Polarization (MDP) achievable for possible observation to perform with the optic systems of POLARIX and IXO we can see that the threshold to detect some degree of polarization is very low. Moreover the closer sources can be resolved to study the flow dynamics and the configuration of the magnetic field. As we can see in Figure 4 a pathfinder mission as POLARIX is able to resolve the main structures in the Crab Nebula. Instead a complete mapping could be performed thanks to the higher resolution of IXO. 


\begin{tabular}{|cc|cc|}
\hline POLARIX & 100 ks observation & IXO & 10 ks observation \\
\hline \hline Flux (mCrab) & MDP $(\%)$ & Flux (mCrab) & MDP $(\%)$ \\
\hline \hline 1000 & $0.39 \%$ & 1000 & $0.27 \%$ \\
\hline 100 & $1.25 \%$ & 100 & $0.86 \%$ \\
\hline 10 & $3.96 \%$ & 10 & $2.73 \%$ \\
\hline
\end{tabular}

Table 2: MDP achievable for possible observations to perform with the optic systems of POLARIX and IXO depending on flux and time integration in the energy band $2 \div 10 \mathrm{keV}$. $\left[1000 \mathrm{mCrab}=2.3 \times 10^{-8} \mathrm{erg} \mathrm{cm}^{-2} \mathrm{~s}^{-1}\right]$

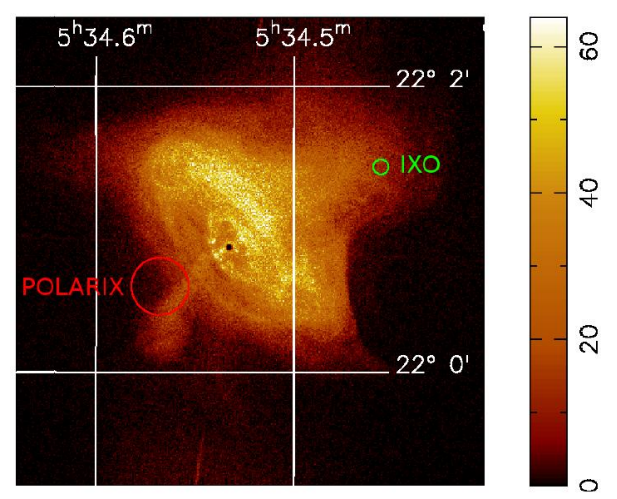

Figure 4: Crab image in X band with overlapped the resolution circles of POLARIX and IXO optics. The diameter of the circles is the HPW.

\section{References}

[1] M. C Weisskopf, J. J. Hester, A. F. Tennant, R. F. Elsner, N. S. Schulz, H. L. Marshall, M. Karovska, J. S. Nichols, D. A. Swartz, J. J. Kolodziejczak, S. L. O’Dell, Discovery of spatial and spectral structure in the X-ray emission from the Crab Nebula, ApJ 536 (L81) 2000

[2] M. C. Weisskopf, E. H. Silver, H. L. Kestenbaum, K. S. Long, R. Novick, A precision measurement of the X-ray polarization of the Crab Nebula without pulsar contamination, ApJ 220 (L117) 1978

[3] N. Bucciantini, L. del Zanna, E. Amato, D. Volpi, Polarization in the inner region of pulsar wind nebulae, A\&A 443(519) 2005

[4] L. Del Zanna, D. Volpi, E. Amato, N. Bucciantini, Simulated synchrotron emission from pulsar wind nebulae, A\&A 453(621) 2006

[5] Y. Nakamura, S. Shibata, Polarization of the Crab Nebula with disordered magnetic components, MNRAS 381 (1489) 2007

[6] R. Bellazzini, G. Spandre, M. Minuti, L. Baldini, A. Brez, L. Latronico, N. Omodei, M. Razzano, M. M. Massai, M. Pesce-Rollins, C. Sgró, E. Costa, P. Soffitta, H. Sipila, E. Lempinen, A sealed Gas Pixel Detector for X-ray astronomy, NIMPA 579 (853) 2007 